\title{
Maximizing post-stroke upper limb rehabilitation using a novel telerehabilitation interactive virtual reality system in the patient's home: study protocol of a randomized clinical trial
}

\author{
Dahlia Kairy ${ }^{\mathrm{a}, *}$, Mirella Veras ${ }^{\mathrm{a}}$, Philippe Archambault ${ }^{\mathrm{b}}$, Alejandro Hernandez ${ }^{\mathrm{c}}$, Johanne Higgins ${ }^{\mathrm{a}}$, \\ Mindy F. Levin ${ }^{\mathrm{b}}$, Lise Poissant ${ }^{\mathrm{a}}$, Amir Raz ${ }^{\mathrm{d}}$, Franceen Kaizer ${ }^{\mathrm{e}}$ \\ a École de réadaptation, Faculté de Médecine, Université de Montréal; CRIR CIUSSS du Centre-Sud-de-lîle-de-Montréal-Institut de réadaptation Gingras-Lindsay de Montréal site, Canada \\ b School of Physical and Occupational Therapy, McGill University; CRIR CISSS de Laval Jewish Rehabilitation Hospital Site, Canada \\ c CRIR CIUSSS du Centre-Sud-de-lîle-de-Montréal-Institut de réadaptation Gingras-Lindsay de Montréal site, Canada \\ d McGill University, Canada \\ e CISSS de Laval, Jewish Rehabilitation Hospital Site, Canada
}

\section{A R T I C L E I N F O}

\section{Article history:}

Received 8 September 2015

Received in revised form 25 November 2015

Accepted 3 December 2015

Available online 4 December 2015

\section{Keywords:}

Telerehabilitation

Stroke

Virtual reality

Protocol

Clinical

Trial

Neurorehabilitaiton

\begin{abstract}
A B S T R A C T
Background: Telerehabilitation (TR), or the provision of rehabilitation services from a distance using telecommunication tools such as the Internet, can contribute to ensure that patients receive the best care at the right time. This study aims to assess the effect of an interactive virtual reality (VR) system that allows ongoing rehabilitation of the upper extremity (UE) following a stroke, while the person is in their own home, with offline monitoring and feedback from a therapist at a distance.

Methods/design: A single-blind (evaluator is blind to group assignment) two-arm randomized controlled trial is proposed, with participants who have had a stroke and are no longer receiving rehabilitation services randomly allocated to: (1) 4-week written home exercise program, i.e. usual care discharge home program or (2) a 4-week home-based TR exercise program using VR in addition to usual care i.e. treatment group. Motor recovery of the UE will be assessed using the Fugl-Meyer Assessment-UE and the Box and Block tests. To determine the efficacy of the system in terms of functional recovery, the Motor Activity Log, a self-reported measure of UE use will be used. Impact on quality of life will be determined using the Stroke Impact Scale-16. Lastly, a preliminary costeffectiveness analysis will be conducted using costs and outcomes for all groups.

Discussion: Findings will contribute to evidence regarding the use of TR and VR to provide stroke rehabilitation services from a distance. This approach can enhance continuity of care once patients are discharged from rehabilitation, in order to maximize their recovery beyond the current available services.
\end{abstract}

(c) 2015 Elsevier Inc. All rights reserved.

\section{Background}

Stroke is a leading cause of death and long-term disability worldwide [1,2] and its incidence is on the rise [2]. Importantly, loss of arm function occurs in up to $85 \%$ of stroke survivors [3], with a significant long-term impact on activities of daily living, leisure activities and work. Recent evidence suggests that home-based telerehabilitation (TR) is a viable approach for upper extremity (UE) training poststroke when rehabilitation services are not available [4]. The Canadian Stroke Best Practice Recommendations, which were updated in 2013, endorse home-based patient monitoring be used when frequent monitoring is needed and face-to-face visits are not available [5].

* Corresponding author at: École de réadaptation, Faculté de Médecine, Université de Montréal, CRIR site Institut de réadaptation Gingras-Lindsay de Montréal, 6300, avenue Darlington, Montréal, Quebec H3S 2J4, Canada.
Promising innovative rehabilitation treatment strategies using TR as well as virtual reality (VR) are emerging with the advent and increased affordability of telecommunication and interactive technologies. VR, an "interface that allows the user to 'interact' with and become 'immersed' in a computer-generated environment in a naturalistic fashion" [6] is increasingly being used in rehabilitation to allow patients to engage in stimulating, repetitive activities [7]. Several reviews and metaanalyses examining the use of movement-based VR for post-stroke rehabilitation, focusing on or including UE rehabilitation, show promising indications for the use of VR, when combined with traditional rehabilitation, to improve recovery post-stroke [8-14] Furthermore, by providing engaging and meaningful activities, VR may motivate patients to continue rehabilitation to maximize functional recovery [10,12]. Several studies have used VR-based applications delivered through TR, using real-time videoconsulting [15-18]. For example, using a VR-based TR system, therapists can monitor the patient's performance using live videoconferencing in order to adjust the patient's activities. Piron et al. 
reported greater improvement in motor performance of the UE after a 4-week training program as compared to usual care [15]. However, real-time communication between the therapist and the patient was required. TR systems can also work offline or asynchronously, with the clinician reviewing the patient's progress reports and making changes to the treatment, in between unsupervised sessions. Integrating off-line monitoring into VR-based TR systems could allow stroke survivors to continue to train and progress their training at home without real-time therapist supervision, thereby providing the opportunity for greater intensity and duration of home-based rehabilitation with minimal additional resources, in order to achieve optimal functional recovery.

Hence, we propose to examine the use of a TR system that allows UE rehabilitation with ongoing off-line monitoring, to be used after usual post-stroke rehabilitation is completed and services are no longer offered. More specifically, the primary objective of the proposed study is to assess the efficacy, in terms of motor recovery, of a novel, patientcentered home-based tailored TR program using an affordable virtual reality (VR) gaming system for UE rehabilitation post-stroke compared to usual care. ${ }^{1}$ Secondary objectives are to assess the impact of the TR VR-based system in terms of function, quality of life, compliance, safety and cost. Such a system, combined with remote off-line monitoring requiring minimal additional resources could allow patients to take charge and pursue their rehabilitation beyond current services, maximizing their potential for recovery.

\section{Methods}

\subsection{Study design}

A single-blind (evaluator is blind to group assignment) two-arm randomized controlled trial (RCT) is proposed for this study [17] with participants who have had a stroke and are no longer receiving rehabilitation services randomly allocated to: (1) a 4-week training program with a home-based TR system (see intervention below), i.e. treatment group or (2) a 4-week written home exercise program, i.e. usual care group.

\subsection{Participants and participant recruitment}

The population of interest is people who have had a stroke and are no longer receiving rehabilitation services but remain with UE motor deficits. Fifty-two people who have sustained a stroke will be recruited and randomly allocated to either the treatment $(n=26)$ or the usual care group $(\mathrm{n}=26)$. Inclusion criteria are: (1) having had an ischemic or hemorrhagic stroke; (2) having residual mild to moderate UE impairment (score 2-6 on the Chedoke-McMaster arm component [18], as long as games can be played); (3) a minimum score of 20 in the Montreal Cognitive Assessment (MoCA); (4) no longer receiving any in- or outpatient rehabilitation services. Exclusion criteria are the factors that may limit participant use of the gaming system or understanding of instructions, as determined by the participant's treating therapist or doctor, a caregiver, the participant themselves or the study coordinator during the recruitment information sessions during which participants can try out the video-game based system. These include: (1) being medically unstable; (2) having severe communication deficits; (3) having visual impairments; (4) having severe balance deficits limiting sitting safely independently; (5) having shoulder pain; (6) having previous UE impairment limiting potential recovery.

Participants will be recruited from rehabilitation centers (previously discharged) and community centers across the Montreal area. Once

\footnotetext{
1 Note: TR is the method for rehabilitation service delivery. VR is the intervention tool used. In the proposed study, a VR system is used in a TR context, with the patient performance data stored and examined by a therapist at a later time, allowing remote monitoring.
}

eligibility is assessed and participants have signed the informed consent form, they will undergo the first evaluation and will be randomly assigned either to the intervention or usual care group. Random allocation will be established by computer-generated random numbers in blocks of six (three usual care participants and three intervention participants), with the resulting list kept securely by a person who is not member of the research team and then put into opaque envelopes that are numbered sequentially. A sample size of 26 participants per group has been determined using $G^{*}$ Power 3.1.3. This was calculated for repeated measures ANOVAs (with one between and one withinsubject factor), assuming a small to medium effect size of 0.2 [19] which was reported in a two-arm RCT with chronic stroke participants [20] using the FMA-UE, our primary outcome measure, and accounting for $20 \%$ attrition $(\alpha=0.05$, power $=0.80)$.

\subsection{Description of the telerehabilitation virtual reality system}

For the treatment group, we propose to use the Jintronix system, which provides a TR platform for UE rehabilitation using VR. This interactive videogame has been in development by Jintronix Inc. (Montreal, Canada) since 2010. The Jintronix System uses the Microsoft Kinect II for Windows technology (Microsoft, Redmond USA) to allow repeated unilateral and bilateral UE training, at customizable difficulty levels. Using Kinect, the Jintronix system can track, in real time, the person's head, arm, trunk and lower body movements when sitting or standing without any sensors. It offers a choice of five UE activities performed against gravity: (1) tracing a horizontally or vertically oriented path; (2) reaching for a target; (3) moving the hands together to catch, carry and drop objects; (4) clapping both hands to catch an object between the two hands; (5) selecting and moving kitchen objects. The games do not require or take into account finger movements. Hence virtual objects are grasped and released when the hand approaches them or moves away from them, and participants are not required to open or close their hands. Objects of Performance on each activity is recorded allowing therapists to review the patient's performance at a later time and to adjust the training program using a clinician portal website (see Fig. 1). All activities are done while sitting.

At the patient's home, the Jintronix system is set up using a regular desktop computer with Windows 8 and a Kinect II motion capture sensor which functions on high-speed Internet. For this study, an internet connection was provided via mobile internet cards using wireless technology, The company has collaborated closely with rehabilitation researchers, clinicians and patients to develop this system. Previous pilot studies by members of the team have shown that the Kinect II camera can reliably track arm movements and have provided data on clinicians' and users' perception of its relevance and potential usefulness for rehabilitation [21]. Previous studies by our team have shown high patient and clinician acceptance [21] as well as preliminary evidence of efficacy and safety when used in a controlled rehabilitation environment [22].

\subsection{Description of the VR-TR interventions}

Participants assigned to the treatment group will meet with the study therapist after their initial evaluation. The therapist will demonstrate the Jintronix system and give them 30 min to try it out, at which time a home-exercise program of about $30 \mathrm{~min}$ will be designed for the participant. The therapist will determine the difficulty (speed, target size, precision, predictability) and choice of games based on the participant's abilities, interests, motivation and fatigue. Once programmed, a date to set up a home system will be determined and participants will then be asked to use the system at least 6 times per week for at least 30 min a day, for 4 weeks. Participants will go through the exercise program that has been preprogrammed by their therapist, although they can choose to spend more (i.e. repeat the program) or less (i.e. stop playing) time using the games. Participant performance will be logged by the gaming system and monitored off-line by the 


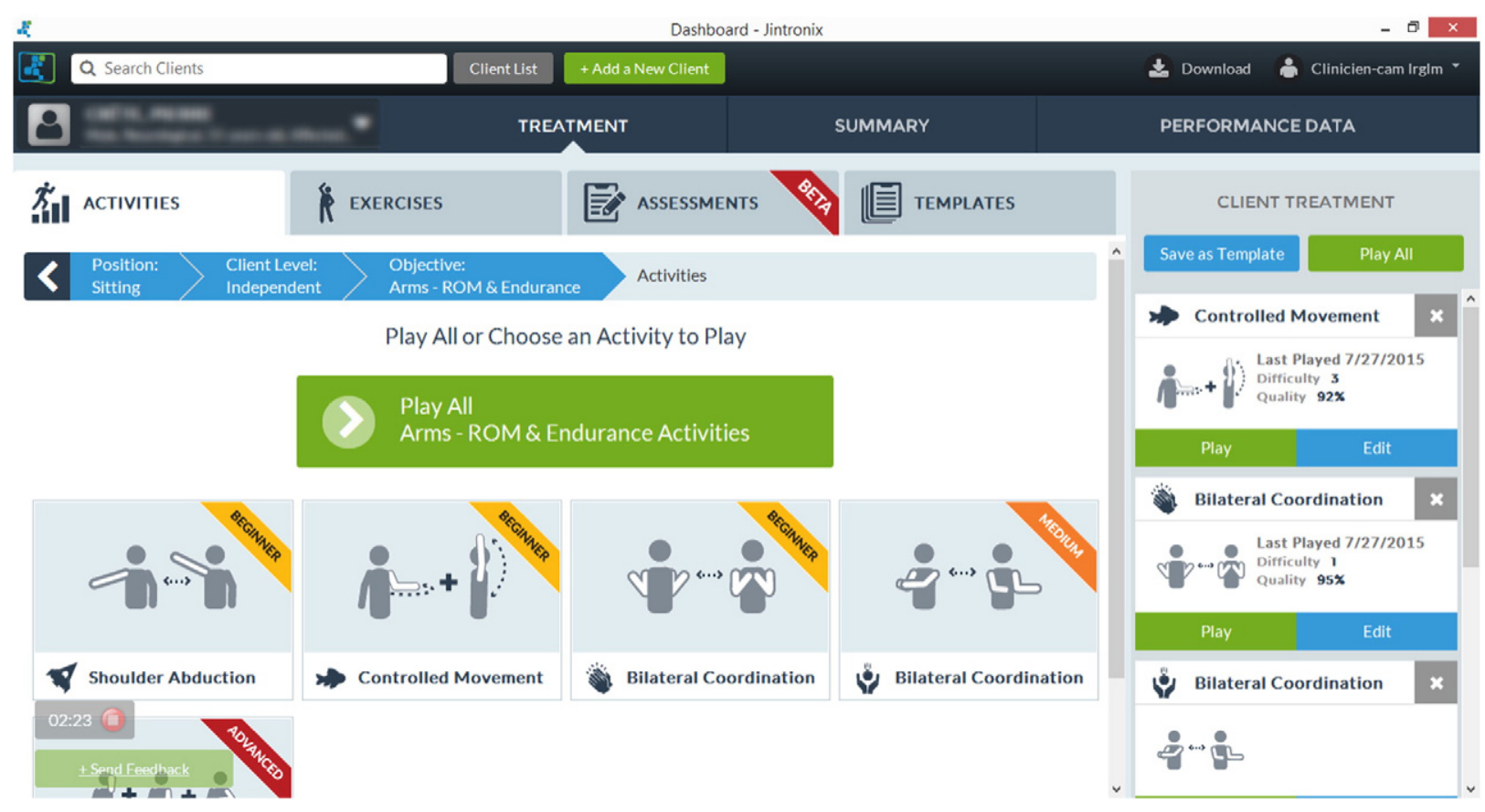

Fig. 1. Screen shot of clinician portal to monitor and adjust patient's exercise program.

therapist. The therapist will tailor the exercise program weekly to ensure the exercises match the person's abilities while remaining motivating and challenging. Throughout the study, participants will be able to contact the study coordinator if they have questions or issues. If the therapist and study coordinator see that the participant is not using the system, the study coordinator will contact them to ensure that there are no technical issues. However, there are no scheduled oneon-one follow-ups with the therapist as this would increase the required resources, and hence likely limiting the generalizability of the intervention. We estimate that at most 15 min per participant per week will be required for the therapist to review and adjust the participant's program.

\subsection{Control group intervention}

In determining the usual care intervention, we took into account that services received after rehabilitation vary greatly, but, at most, patients who have completed their rehabilitation in the Montreal area receive at discharge a home exercise program, such as the GRASP, a standardized validated post-stroke upper extremity exercise program [23], as per current stroke guidelines. Therefore participants in the control group will be asked to continue any previously recommended exercise program or if none was provided, they will be provided with the GRASP and asked to perform it 6 days a week for $30 \mathrm{~min}$. Similarly, most participants in the intervention group will have also been prescribed a home exercise program at discharge from rehabilitation. For those who were not prescribed a program, the GRAPS will be provided so as to ensure all participants receive similar usual care recommendations, hence reducing the heterogeneity among participants.

\subsection{Data collection and study outcomes}

Study participant characteristics will be documented at baseline for both groups (age, gender, handedness, stroke characteristics, level of disability, services received, comorbidities using the Cumulative Illness Rating Scale $[24,25])$. Outcome measures will be documented at the start (T0), end (T1) and one month after the 4-week intervention (T2), and all evaluation sessions will take place at the research center. Study evaluators will be trained for all assessments and blinded as to group assignment. Evaluators will not be involved in any other aspect of the study and are told not to discuss group assignment with participants. Participants are also told not to discuss it with their evaluators. Given the nature of the intervention, therapists and participants cannot be masked.

The primary outcome for this study is the Fugl-Meyer AssessmentUE [26] (FMA-UE), a measure of UE motor impairment. The FMA-UE, a performance-based measure of UE impairment used in research to describe motor recovery, has been shown to have excellent reliability including internal consistency, inter-rater reliability, intra-rater reliability and test-retest reliability post-stroke [27]. A secondary outcome measure of UE impairment is the Box and Blocks test [28]. The Box and Blocks test is a measure of gross motor dexterity that has been shown to have high test-retest reliability [29], construct validity [30] and concurrent validity with the Functional Independence Measure [31].It is also essential to document the impact of the training on UE use in daily activities [32]. To determine the efficacy of the training in terms of functional recovery, the Motor Activity Log [32-35], a selfreported measure of UE use will be used. This rates the quality and frequency of use of the UE in 28 everyday tasks, and is administered by interview. It has been shown to have high internal consistency [34], construct validity, and good test-retest reliability [26]. Impact on quality of life will be determined using the Stroke Impact Scale-16 [36], a stroke-specific, self-reported, health status measure containing 16 items capturing daily activities. It has been shown to have good internal consistency as well as convergent and discriminant validity [37]. Both the treatment and usual care groups will use a log to document self-reported time spent exercising (i.e. compliance), motivation, appreciation, other services received and adverse events such as discomfort and oedema of the UE. The actual amount of time spent playing the VR games will be recorded directly from the Jintronix system.

\subsection{Economic Evaluation}

A preliminary economic evaluation (cost effectiveness) will be conducted using costs and outcomes for all groups according to intention to treat principle. This type of analysis can be used to compare programs which have a common effect [34]. Costs for each approach will be recorded from the perspective of the organization compared to 
change in FMA-UE, the primary outcome, using an incremental costeffectiveness ratio (ICER), which documents the cost per unit of outcome for each group. The following costs will be included: clinician services costs (intervention group only: physiotherapist time planning the session, intervention time and time spent to trouble shoot (calls, etc.), technician services (intervention group only: distance and time traveled for installation and for trouble shooting, time spent for technical problem resolution), equipment cost (both groups) and internet costs (intervention group only). ICER will be calculated by using the mean for both cost and effectiveness measures and sensitivity analysis will be performed on the most important assumptions in order to assess the uncertainty of the results [38].

\subsection{Statistical analyses}

All statistical analyses will be performed using SAS 9.3 (SAS Institute Inc., Cary, NC, USA) statistical software. Descriptive statistics will be used to highlight main demographic characteristics of participants in both groups (averages and standard deviations for continuous variables, frequencies and percentages for categorical variables). To address, the study's primary and secondary objectives two-way repeated measure ANOVA mixed-model approach will be used, for all outcomes measures, with one between subject factor (group), one within subject factor (time), as well as covariates to account for variables such as age, severity of stroke or other variables that may impact outcome or may differ between groups at baseline. Residual plots will be inspected to verify linearity, normality and homoscedasticity assumptions for all models as well as to identify potential influential outliers. Pre-planned specific pairwise comparisons between groups for each time will be conducted when appropriate. For all inferential analyses the probability of type 1 error is a-priori fixed at alpha $=0.05$ and will include reporting the 95\% Confidence Interval for each estimate.

\section{Discussion}

Findings will contribute to evidence regarding the use of TR and VR to provide rehabilitation services from a distance, using a service delivery model not requiring one-on-one direct supervision in the chronic stages post-stroke. In addition, this TR-VR intervention uses readily available equipment and internet access, increasing the likelihood that this type of model of service delivery can in fact be implemented. Such an approach can enhance continuity of care once patients are discharged from rehabilitation, ensuring they continue to improve their recovery beyond what is currently available. It is also a relevant service delivery option for more rural and remote areas where access to out-patient services is often more limited [5]. This collaborative initiative includes researchers in neurorehabilitation ( $\mathrm{JH}, \mathrm{MFL})$, rehabilitation technologies (DK, PA, MFL), cognitive sciences (AR), information systems and knowledge translation (DK, LP, PA) and a decision maker in rehabilitation (FK). The active participation in the research team of researchers from two main rehabilitation centers in the Montreal and Laval areas will help facilitate future implementation of this service delivery method in the clinical setting [20,39-41].

Furthermore, ongoing development of the Jintronix system with Jintronix Inc. will allow for additional rehabilitation protocols to be developed, hence creating opportunities for ongoing rehabilitation in areas such as balance and lower extremity rehabilitation. While findings from this study may not be generalized to other patient populations, home programs using such TR and VR applications, with occasional clinician monitoring, are equally relevant for other patient populations, in particular for individuals with chronic conditions causing long-term disability who could benefit from long-term disease management tools without requiring substantial resources.

This study will provide evidence for future guideline reviews regarding the effectiveness of such TR interventions to provide UE post-stroke rehabilitation beyond what current services allow for. Furthermore, this is a relatively affordable VR technology, using commonly available internet connections and computers, hence reducing the financial barriers related to previous technologies used for TR or VR.

\section{Conflict of interest}

Jintronix provided licenses for use of their gaming system, and provided technical support and servers. We declare that we have no conflicts of interest in the authorship or publication of this study.

\section{Acknowledgments}

The proposed project is funded by the Heart and Stroke Foundation of Canada and Canadian Partnership for Stroke Research. We are grateful to Mark Evin and the Jintronix Inc. team, for the ongoing development of the system and technical support for this project.

\section{References}

[1] V.L. Feigin, C.M. Lawes, D.A. Bennett, S.L. Barker-Collo, V. Parago, Worldwide stroke incidence and early case fatality reported in 56 population-based studies: a systematic review, Lancet Neurol. 8 (4) (2009) 355-369.

[2] V.L. Roger, A.S. Go, D.M. Lloyd-Jones, ea. Heart disease and stroke statistics-2011 update: a report from the American Heart Association, Circulation 123 (4) (2011) e18-e209.

[3] D.S. Nichols-Larsen, P.C. Clark, A. Zeringue, A. Greenspan, S. Blanton, Factors influencing stroke survivors' quality of life during subacute recovery, Stroke 36 (7) (2005) 1480-1484.

[4] J. Langan, K. Delave, L. Phillips, P. Pangilinan, S.H. Brown, Home-based telerehabilitation shows improved upper limb function in adults with chronic stroke: a pilot study, J. Rehabil. Med. 45 (2) (2013) 217-220.

[5] A. Dawson, K.J. MA, N. Foley, R. Teasell, Chapter 5: stroke rehabilitation, in: M.P.G.G.B.M. Lindsay, S. Phillips (Eds.), Canadian Best Practice Recommendations for Stroke Care, Canadian Stroke Best Practices and Standards Working Group, 2013.

[6] K. Laver, S. George, S. Thomas, J. Deutsch, M. Crotty, Virtual reality for stroke rehabilitation, Cochrane Database Syst. Rev. 9 (2011).

[7] G.C. Burdea, Virtual rehabilitation-benefits and challenges, Methods Inf. Med. 42 (5) (2003) 519-523.

[8] M. Cameirao, S. Bermudez i Badia, P. Vershure, Virtual reality based upper extremety rehabilitation following stroke: a review, J. Cyber Ther. Rehabil 1 (1) (2008) 63-74.

[9] J.H. Crosbie, S. Lennon, J.R. Basford, S.M. McDonough, Virtual reality in stroke rehabilitation: still more virtual than real, Disabil. Rehabil. 29 (14) (2007) 1139-1146 (discussion 1147-1152).

[10] B. Lange, S.M. Flynn, A.A. Rizzo, Game-based telerehabilitation, Eur. J. Phys. Rehabil. Med. 45 (1) (2009) 143-151.

[11] G. Saposnik, M. Levin, Virtual reality in stroke rehabilitation: a meta-analysis and implications for clinicians, Stroke 42 (5) (2011) 1380-1386.

[12] D. Celinder, H. Peoples, Stroke patients' experiences with Wii Sports ${ }^{\circ}$ during inpatient rehabilitation, Scand. J. Occup. Ther. 19 (5) (2012) 457-463.

[13] B.C. Huijgen, M.M. Vollenbroek-Hutten, M. Zampolini, et al., Feasibility of a homebased telerehabilitation system compared to usual care: arm/hand function in patients with stroke, traumatic brain injury and multiple sclerosis, J. Telemed. Telecare 14 (5) (2008) 249-256.

[14] L. Piron, P. Tonin, A.M. Atzori, et al., Virtual environment system for motor telerehabilitation, Stud. Health Technol. Inform. 85 (355) (2002) 361

[15] L. Piron, P. Tonin, E. Trivello, L. Battistin, M. Dam, Motor tele-rehabilitation in poststroke patients, Med. Inform. Internet Med. 29 (2) (2004) 119-125.

[16] L. Piron, A. Turolla, M. Agostini, et al., Exercises for paretic upper limb after stroke: a combined virtual-reality and telemedicine approach, J. Rehabil. Med. 41 (12) (2009) 1016-1102.

[17] K.F. Schulz, D.G. Altman, D. Moher, CONSORT 2010 statement: updated guidelines for reporting parallel group randomised trials, BMJ 340 (2010) c322.

[18] C. Gowland, P. Stratford, M. Ward, et al., Measuring physical impairment and disability with the Chedoke-McMaster stroke assessment, Stroke 24 (1) (1993) 58-63.

[19] F. Faul, E. Erdfelder, A. Lang, A. Buchner, G*Power 3: a flexible statistical power analysis program for the social, behavioral, and biomedical sciences, Behav. Res. Methods 39 (2) (May 2007) 175-191.

[20] M. Pang, J. Harris, J. Eng, A community-based upper-extremity group exercise program improves motor function and performance of functional activities in chronic stroke: a randomized controlled trial, Arch. Phys. Med. Rehabil. 87 (1) (2006) 1-9.

[21] P. Archambault, N. Norouzi, D. Kairy, J. Solomon, M. Levin, Towards establishing Clinical Guidelines for an Arm Rehabilitation Virtual Reality System, Replace, Repair, Restore, Relieve - Bridging Clinical and Engineering Solutions in NeuroRehabilitation, 7(263) 2014, p. 270

[22] D. Kairy, L. Poissant, J. Higgins, A. Hernandez, P. Archamault, N. Norouzi-Gheidari, Using a virtual reality gaming system to supplement upper extremity rehabilitation post stroke. 92nd Annual Conference of the American Congress of Rehabilitation Medicine Dallas Texas, October 25-30 2015

[23] J.E. Harris, J.J. Eng, W.C. Miller, A. Dawson, A self-administered graded repetitive arm supplementary program (GRASP) improves arm function during inpatient stroke 
rehabilitation: a multi-site randomized controlled trial, Stroke 40 (6) (2009) 2123-2128.

[24] S. Giaquinto, Comorbidity in post-stroke rehabilitation, Eur. J. Neurol. 10 (3) (May 2003) 235-238.

[25] B. Linn, M. Linn, L. Gurel, Cumulative illness rating scale, J. Am. Geriatr. Soc. 16 (5) (1968) 622-626.

[26] A.R. Fugl-Meyer, L. Jaasko, I. Leyman, S. Olsson, S. Steglind, The post-stroke hemiplegic patient. 1. A method for evaluation of physical performance, Scand. J. Rehabil. Med. 7 (1) (1975) 13-31.

[27] J.H. Lin, I.P. Hsueh, C.F. Sheu, C.L. Hsieh, Psychometric properties of the sensory scale of the Fugl-Meyer assessment in stroke patients, Clin. Rehabil. 18 (4) (2004) 391-397.

[28] V. Mathiowetz, G. Volland, N. Kashman, K. Weber, Adult norms for the box and block test of manual dexterity, Am. J. Occup. Ther. 39 (6) (1985) 386-391.

[29] F. Cromwell, Occupational Therapist's Manual for Basic Skill Assessment: Primary Prevocational Evaluation, Altadena, Fair Oaks Printing, 1976.

[30] J. Desrosiers, G. Bravo, R. Hebert, E. Dutil, L. Mercier, Validation of the Box and Block Test as a measure of dexterity of elderly people: reliability, validity, and norms studies, Arch. Phys. Med. Rehabil. 75 (1994) 751-755 (1994;75:751-5).

[31] R. Hebert, R. Carrier, A. Bilodeau, The functional autonomy measurement system (SMAF): description and validation of an instrument for the measurement of handicaps, Age Ageing 17 (1988) 293-302.

[32] G. Uswatte, E. Taub, D. Morris, K. Light, P.A. Thompson, The motor activity log-28: assessing daily use of the hemiparetic arm after stroke, Neurology 67 (7) (2006) 1189-1194.
[33] G. Uswatte, E. Taub, Implications of the learned nonuse formulation for measuring rehabilitation outcomes: lessons from constraint-induced movement therapy, Rehabil. Psychol. 50 (1) (2005) 34-42.

[34] G. Uswatte, E. Taub, D. Morris, M. Vignolo, McCulloch. Reliability and validity of the upperextremity motor activity log-14 for measuring real-world arm use, Stroke 36 (11) (2005) 2493-2496.

[35] S. Ashford, M. Slade, F. Malaprade, L. Turner-Stokes, Evaluation of functional outcome measures for the hemiparetic upper limb: a systematic review, J. Rehabil. Med. 40 (10) (Nov 2008) 787-795.

[36] P.W. Duncan, S.M. Lai, R.K. Bode, S. Perera, J. DeRosa, Stroke impact scale-16: a brief assessment of physical function, Neurology 60 (2) (2003) 291-296.

[37] B. Edwards, B. O'Connell, Internal consistency and validity of the stroke impact scale 2.0 (SIS 2.0) and SIS-16 in an Australian sample, Qual. Life Res. 12 (8) (2003) 1127-1135.

[38] J. Polisena, S. Tam, A. Lodha, A. Laporte, P.C. Coyte, W.J. Ungar, An economic evaluation of asthma action plans for children with asthma, J. Asthma 44 (7) (2007 Sep) 501-508.

[39] M. Drummond, Methods for the Economic Evaluation of Health Care Programmes, Oxford University Press, New York, 1997.

[40] D. Raphael, Barriers to addressing the societal determinants of health: public health units and poverty in Ontario, Canada, Health Promot. Int. 18 (4) (2003 Dec 1) 397-405.

[41] E. Rogers, Diffusion of Innovations, fourth ed. The Free Press, New York, 1995. 